\title{
POLYNOMIAL VALUES, THE LINKING FORM AND UNKNOTTING NUMBERS
}

\author{
A. Stoimenow
}

\begin{abstract}
We show how the signed evaluations of link polynomials can be used to calculate unknotting numbers. We use the Jones-Rong value of the BrandtLickorish-Millett-Ho polynomial $Q$ to calculate the unknotting numbers of 816 , 949 and 6 further new entries in Kawauchi's tables. Another method is developed by applying and extending the linking form criterion of Lickorish. This leads to several conjectured relations between the Jones-Rong value of $Q$ and the linking form.
\end{abstract}

\section{Introduction}

The unknotting number $u(K)$ of a knot $K$ is defined as the minimal number of crossing changes in any diagram of $K$ needed to turn $K$ into the unknot (see e.g. [A, Km, Ko, Na, Sc, Ta, We, Zh]). Despite its simple definition, the unknotting number has proved hard to calculate. The tables in $[\mathrm{Kw}]$ show that, after several decades of work, the unknotting number of each fifth prime knot with 10 or fewer crossings remains unknown.

In this paper, we develop two at first glance different approaches to the calculation of unknotting numbers - using the evaluations of the link polynomials [J, LM, Lp, Rn] and the linking form $\lambda$ on $H_{1}\left(D_{K}\right)$ [Li].

As outcome, we settle the problem of determining the unknotting number for 9 (that is, about one sixth) of the open nine and ten crossing knots in Kawauchi's tables $[\mathrm{Kw}]$. The table below summarizes these examples:

(1)

\begin{tabular}{|c|c|c|c|}
\hline method & $u=1$ & $u=2$ & $u=3$ \\
\hline $\begin{array}{c}\text { observation } \\
Q\end{array}$ & $10_{131}$ & 121 & $0_{1}$ \\
\hline$\lambda$ & & $8_{16}, 10_{86}, 10_{105}, 10_{106}, 10_{109}, 10_{116}, 10_{121}$ & $9_{49}$ \\
\hline
\end{tabular}

Received October 16, 2003.

2000 Mathematics Subject Classification. 57M25 (primary), 57M12 (secondary).

Key words and phrases. Jones polynomial, Goeritz matrix, double branched cover, linking form, Brandt-Lickorish-Millett-Ho polynomial, unknotting number, signature.

Supported by a DFG postdoc grant. 
Our criteria can also be applied to some simple composite knots (we give a table of such unknotting numbers in an appendix) and to knot distance [Mr].

It is striking that the outcome of both methods - the $Q$ polynomial and the linking form - give surprisingly similar, although not identical, results. This is clearly hardly a matter of accidental coincidence, and thus we are led to several conjectures on relations between both. We will mention the evidence for most of these conjectures while discussing the various examples, and explicitly compile the conjectures for the benefit of the reader at the end of the paper, hoping to motivate further investigations on this subject.

\section{Preliminaries and notation}

For each knot $K$ we have a sequence of knots $K_{i}$

$$
K_{0} \rightarrow K_{1} \rightarrow K_{2} \rightarrow \cdots \rightarrow K_{n}
$$

such that $K=K_{0}, K_{n}$ is the unknot, and $K_{i}$ differs from $K_{i-1}$ only by a crossing change. We call a sequence (2) an unknotting sequence for $K$. The minimal length $n$ of an unknotting sequence (2) for $K$ is the unknotting number $u(K)$ of $K$.

Henceforth, $D_{K}$ denotes the double branched cover of $S^{3}$ over a knot $K$. By $H_{1}=H_{1}\left(D_{K}\right)=H_{1}\left(D_{K}, \mathbb{Z}\right)$ we denote its homology group over $\mathbb{Z}$. (The various abbreviated versions will be used at places where no confusion arises; $H_{1}$ will be used throughout the paper only in this context, so that, for example, when we talk of $H_{1}$ of a knot, always $H_{1}$ of its double cover will be meant.) $H_{1}$ is a finite commutative group of odd order. This order is called the determinant of a knot $K$, and it will be denoted as $\operatorname{det}(K)$. (This generalizes to links $L$, by putting $\operatorname{det}(L)=0$ to stand for infinite $H_{1}\left(D_{L}\right)$.) By the classification of finite commutative groups, $H_{1}$ decomposes into a direct sum of finite (odd order) cyclic groups $\mathbb{Z}_{p}$; their orders are called torsion numbers. Wendt [We] proved that $u(K)$ is not smaller than the number of torsion numbers of $H_{1}\left(D_{K}\right)$. The group $H_{1}$ is also equipped with a bilinear form $\lambda: H_{1} \times H_{1} \rightarrow \mathbb{Q} / \mathbb{Z}$, called the linking form (see [Li, MY] for example).

In the following knots and links will be assumed oriented, but sometimes orientation will be irrelevant.

The Jones polynomial $V$ (introduced in $[\mathrm{J}]$, but now commonly used with the convention of [J3]) is a Laurent polynomial in one variable $t$ of oriented knots and links, and can be defined by being 1 on the unknot and the (skein) relation

$$
t^{-1} V\left(L_{+}\right)-t V\left(L_{-}\right)=-\left(t^{-1 / 2}-t^{1 / 2}\right) V\left(L_{0}\right) .
$$

Herein $L_{ \pm, 0}$ are three links with diagrams differing only near a crossing.
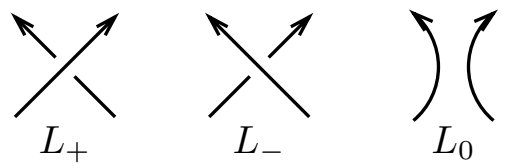
We call the crossings in the first two fragments respectively positive and negative, and a crossing replaced by the third fragment smoothed out. A triple of links that can be represented as $L_{ \pm, 0}$ in (4) is called a skein triple. The sum of the signs $( \pm 1)$ of the crossings of a diagram $D$ is called writhe of $D$ and written $w(D)$.

A different interpretation of the Jones polynomial than via skein rules has been developed by Kauffman [Ka] (see also [Ad, §6.2]). The Kauffman state model is sometimes more useful than the skein approach, and we shall also consider it below. Recall, that the Kauffman bracket $\langle D\rangle$ of a(n unoriented) link diagram $D$ is a Laurent polynomial in a variable $A$, obtained by summing over all states the terms

$$
A^{\# A-\# B}\left(-A^{2}-A^{-2}\right)^{|S|-1} .
$$

Herein a state is a choice of splittings of type $A$ or $B$ for any single crossing (see figure 1), \#A and \#B denote the number of type A (respectively type $\mathrm{B}$ ) splittings and $|S|$ the number of (disjoint) circles obtained after all splittings in a state.

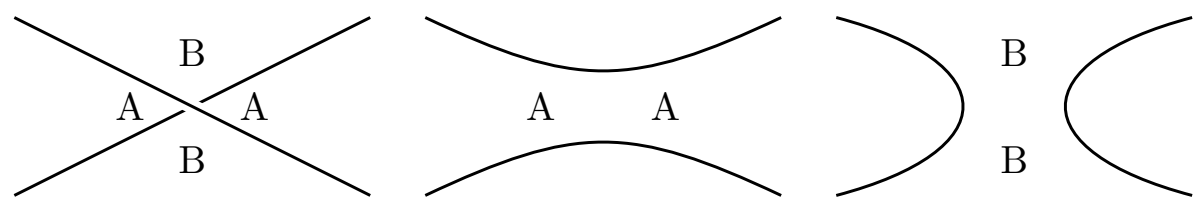

Figure 1: The A- and B-corners of a crossing, and both its splittings. The corner A (respectively $B$ ) is the one passed by the overcrossing strand when rotated counterclockwise (respectively clockwise) towards the undercrossing strand. A type A (respectively B) splitting is obtained by connecting the A (respectively B) corners of the crossing.

The Jones polynomial of a link $L$ can be calculated from the Kauffman bracket, by evaluating the latter on the (unoriented version of) a diagram $D$ of $L$, and then multiplying by a power of $t$ coming from the (orientation dependent) writhe of $D$ :

$$
V_{L}(t)=\left.\left(-t^{-3 / 4}\right)^{-w(D)}\langle D\rangle\right|_{A=t^{-1 / 4}} .
$$

The signature $\sigma$ is a $\mathbb{Z}$-valued invariant of knots and links. Originally it was defined in terms of Seifert matrices [Ro]. We have that $\sigma(L)$ has the opposite parity to the number of components of a link $L$, whenever the determinant of $L$ is non-zero (i.e. $H_{1}\left(D_{L}\right)$ is finite). This in particular always happens for $L$ being a knot, so that $\sigma$ takes only even values on knots.

Most of the early work on the signature was done by Murasugi [Mu], who showed several properties of this invariant. In particular the following property 
is known: if $L_{ \pm, 0}$ form a skein triple, then

$$
\begin{aligned}
& \sigma\left(L_{+}\right)-\sigma\left(L_{-}\right) \in\{0,1,2\}, \\
& \sigma\left(L_{ \pm}\right)-\sigma\left(L_{0}\right) \in\{-1,0,1\} .
\end{aligned}
$$

(Note: In (7) one can also have $\{0,-1,-2\}$ instead of $\{0,1,2\}$, since other authors, like Murasugi, take $\sigma$ to be with opposite sign. Thus (7) not only defines a property, but also specifies our sign convention for $\sigma$.) We remark that for knots in (7) only 0 and 2 can occur on the right. A consequence of this relation is the inequality $u(K) \geq|\sigma(K) / 2|$. After Wendt's inequality, this was one of the first important results on the unknotting number.

Now we introduce the Brandt-Lickorish-Millett-Ho polynomial $Q_{K}=Q_{K}(z)$ of $K$ [BLM, Ho]. Recall, that the $Q$ polynomial is a Laurent polynomial in one variable $z$ for links without orientation, defined by being 1 on the unknot and the relation

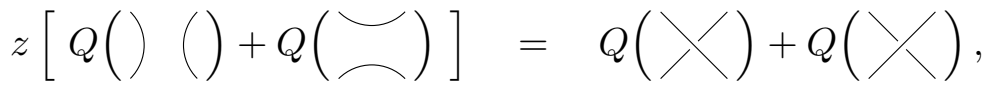

where again the fragments denote link diagrams equal except in the specified fragment.

If we modify the skein relation for $V$ by omitting the coefficients $t^{\mp 1}$ of $L_{ \pm}$ on the left of (3), we obtain the skein relation for another (and more classical) polynomial invariant, the Alexander polynomial $\Delta(t)$ (see [Ro]).

All three polynomials allow one to express the determinant of $K$, as

$$
\operatorname{det}(K)=\left|\Delta_{K}(-1)\right|=\left|V_{K}(-1)\right|=\sqrt{Q_{K}(2)} .
$$

There are further special values of the Jones and $Q$ polynomial, which will be discussed in the following (see $[\mathrm{J} 3, \S 12],[\mathrm{LM}]$ and $[\mathrm{BLM}]$ for more details).

By $k \% 2 \in\{0,1\}$ we denote the parity of $k$ and by $\lfloor k\rfloor$ the greatest integer not exceeding $k$. 'W.l.o.g.' will abbreviate 'without loss of generality'. The notation for knots we use is this of Rolfsen [Ro].

\section{The Jones polynomial}

We first start by a property of the Jones polynomial $V$ of an unknotting number one knot, which slightly generalizes Traczyk's criterion for the unknotting number one case. It is related to the signed unknotting number (see [Tr, CL]).

Proposition 3.1. Let $K$ be an unknotting number one knot which can be unknotted by switching a positive crossing to the negative, and set

$$
\tilde{V}=1-\frac{V_{K}-1}{t-1}
$$

Then there exists a knot $K^{\prime}$ with $V_{K^{\prime}}=t^{-\tilde{V}^{\prime}(1)} \tilde{V}$.

Proof. Let $D$ be the diagram of $K$ unknotting by a change of a positive crossing. (We call this crossing unknotting crossing.) W.l.o.g. we can assume $D$ to have 
zero writhe (add kinks). Thus, considering the Kauffman bracket version of $V$, we have

$$
\begin{aligned}
& \langle>\rangle=V_{K}\left(A^{-4}\right) \text { and } \\
& \langle>\rangle=A^{-6} .
\end{aligned}
$$

Resolving the crossing according to the Kauffman bracket relation, we get

$$
\left(\begin{array}{cc}
A & A^{-1} \\
A^{-1} & A
\end{array}\right)\left(\begin{array}{l}
\langle\zeta\rangle \\
\langle\succ
\end{array}\right)=\left(\begin{array}{c}
V_{K}\left(A^{-4}\right) \\
A^{-6}
\end{array}\right) \text {. }
$$

The sign of the crossing switched shows that from both splicings, is the one that again corresponds to a knot. We find

$$
\begin{aligned}
\langle\supset & =-\frac{V_{K}\left(A^{-4}\right) A^{-1}-A \cdot A^{-6}}{A^{2}-A^{-2}} \\
& =A^{-3}\left[\frac{-A^{-4}+V_{K}\left(A^{-4}\right)}{A^{-4}-1}\right],
\end{aligned}
$$

and setting $t=A^{-4}$ we get

$$
\left\langle\circlearrowright=-A^{-3}\left[1-\frac{V_{K}-1}{t-1}\right]\right. \text {. }
$$

Thus the Jones polynomial of $K^{\prime}=\smile \operatorname{differs}$ from $\tilde{V}$ just by a unit in $Z\left[t, t^{-1}\right]$, and the properties $V_{\tilde{K}}(1)=1$ and $V_{\tilde{K}}^{\prime}(1)=0$ for $\tilde{K}=K, K^{\prime}$ show that this unit is precisely $t^{-\tilde{V}^{\prime}(1)}$.

It has been known for a while, by [LM], that $V\left(e^{\pi i / 3}\right)= \pm(i \sqrt{3})^{d}$ for some $d \in \mathbb{N}$, which was observed by Traczyk in $[\operatorname{Tr}]$ to show $u \geq d$ (a conclusion that alternatively follows when combining [LM] and [We]). He used a more intricate combination of the sign of the evaluation and the signature to show that sometimes $u>d$, proving $u\left(10_{67}\right)=2$. Traczyk's observation for $u=1$ is also contained in ours, but we can now apply more on what we know on the values of $V$. We have the following properties, basically due to Jones [J3, §12] and Lickorish-Millett [LM] (see also [St]).

Proposition 3.2. (Jones $[\mathrm{J} 3, \S 12]$ ) Let $V \in \mathbb{Z}\left[t, t^{-1}\right]$ be the Jones polynomial of a knot. Then $V \equiv 1 \bmod (t-1)\left(t^{3}-1\right)$ and $V \equiv \pm 3^{k}(2 t-1)^{d} \bmod t^{2}-t+1$ for some $k \in \mathbb{N}$ and $d \in\{0,1\}$, such that if $3 \nmid V(-1), k=d=0$ and if $3 \mid V(-1)$, $k+d>0$ and $3^{2 k+d} \mid V(-1)$. Furthermore, we have the Arf invariant identity $V(i)=(-1)^{V^{\prime \prime}(1) / 6}$.

(We reformulated these conditions using the fact, known from standard Galois theory, that the evaluation of a polynomial $P$ in some algebraic number $v$ is equivalent to the residue of $P$ modulo the minimal polynomial of $v$.) 
A more general and simple special case is the following.

Corollary 3.1. Let $K$ be an achiral knot (or weaker a knot with $V_{K}(t)=$ $\left.V_{K}(1 / t)\right)$, and $3 \mid V_{K}(-1)$. Then even $9 \mid V_{K}(-1)$, and $u(K)>1$.

Proof. If $K$ is achiral, then for $|t|=1$ we have $\bar{t}=1 / t$, and thus $V_{K}(t)$ is real. If $u(K)=1$, then $\operatorname{dim}_{\mathbb{Z}_{3}} H_{1}\left(D_{K}, \mathbb{Z}_{3}\right)=1$, but in this case $V_{K}\left(e^{\pi i / 3}\right)= \pm i \sqrt{3}$, which is not real. Therefore, $u(K) \geq \operatorname{dim}_{\mathbb{Z}_{3}} H_{1}\left(D_{K}, \mathbb{Z}_{3}\right) \geq 2$, so in particular $9 \mid V_{K}(-1)$.

Remark 3.1. It was observed in [St, §5], that the property $\left(V_{K}\left(e^{\pi i / 3}\right)\right)^{2}$ $V_{K}(-1)$ for the Jones polynomial $V_{K}$ of a knot $K$ does not (in general) follow from the properties of $V$ listed in [J3, §12].

A similar statement, which is unrelated to unknotting numbers, but worth mentioning, is the following corollary. Here '3-equivalent' means transformable by a sequence of Nakanishi's 3-moves (see [St]):

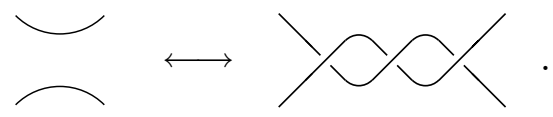

Corollary 3.2. If a knot $K$ is 3-equivalent to an unlink of an even number of components, then $K$ is chiral.

Proof. A 3-move (11), regardless of strand orientation and possible change of the number of components, preserves $\left|V\left(e^{\pi i / 3}\right)\right|$. If now a knot $K$ is 3-equivalent to an unlink of an even number of components, then $\left|V_{K}\left(e^{\pi i / 3}\right)\right|=3^{k} \sqrt{3}$ for some $k \in \mathbb{N}$. Thus in $V_{K}\left(e^{\pi i / 3}\right)= \pm(i \sqrt{3})^{l}$ we must have odd $l$, that is, the value is, up to a sign, an odd power of $i \sqrt{3}$. But then this value is not real, and $V_{K}$ is not self-conjugate.

\section{The $Q$ polynomial}

Now we go a step further from the proof of Proposition 3.1 and consider the $Q$ polynomial [BLM, Ho]. Of main relevance is the result of Jones [J2] and Rong [Rn] on the form $\pm \sqrt{5}^{k}$ of $Q\left(\frac{ \pm \sqrt{5}-1}{2}\right)$ for some $k \in \mathbb{N}$. This quantity is referred to as the Jones-Rong value of $Q$.

Proposition 4.1. Let $K$ be an unknotting number one knot of determinant $\operatorname{det}(K)=2 n+1, n>0$. Set for $k \in \mathbb{N}$

$$
S_{5}(k):= \begin{cases}\{0\} & 5 \nmid k \\ \left\{l>0: 5^{l} \mid k\right\} & 5 \mid k\end{cases}
$$

Then there exist numbers $k \in S_{5}(n)$ and $l \in S_{5}(n+1)$ such that

$$
1+Q_{K}(z) \equiv z\left( \pm_{1} 5^{\lfloor k / 2\rfloor}(2 z+1)^{k \% 2} \pm_{2} 5^{\lfloor l / 2\rfloor}(2 z+1)^{l \% 2}\right) \bmod z^{2}+z-1,
$$

for some (independent) sign choices $\pm_{1,2} \in\{+,-\}$. 
Proof. We return to the arguments in the proof of Proposition 3.1. A similar calculation of \langle)(\rangle shows that it differs from $\langle\underset{\sim}{\longrightarrow}$ just by multiplication and addition of a unit, hence the determinants of $\left.L_{1}=\right) \quad\left(\right.$ and $L_{2}=$ differ just by \pm 1 . Then these determinants are $n$ and $n+1$ respectively (the even one corresponding to the 2 component link and the odd one to the knot). To see this, either evaluate (10) and use the skein relation (3) for $V$ for the unknotting crossing change at $t=-1$, or alternatively use the relation (9) for $Q$ evaluated at $z=2$ and the property of $[\mathrm{BLM}]$ that $\operatorname{det}(K)=\sqrt{Q(2)}$. Then consider the $Q$ relation at $z=\frac{\sqrt{5}-1}{2}$ at the unknotting crossing, and let $k$ and $l$ be the two numbers $\operatorname{dim} H_{1}\left(D_{L_{1,2}}, \mathbb{Z}_{5}\right)$. The claim follows using the Jones-Rong value of $Q$ and rewriting the resulting condition as a congruence modulo the minimal polynomial of $\frac{ \pm \sqrt{5}-1}{2}$.

The criterion for $Q$ can be generalized to higher unknotting numbers.

Theorem 4.1. If $Q_{K}\left(\frac{\sqrt{5}-1}{2}\right)=-(-\sqrt{5})^{k}$, then $u(K)>k$.

Proof. The proof goes through by slight modification of the arguments in the proof of Proposition 4.1, and via induction on the unknotting number, as in [Tr]. One needs to observe, using the relation of $Q$, that if $K$ and $K^{\prime}$ differ by a crossing change, then the value $Q_{K}\left(\frac{\sqrt{5}-1}{2}\right) / Q_{K^{\prime}}\left(\frac{\sqrt{5}-1}{2}\right) \in\left\{ \pm 1,-5^{ \pm 1 / 2}\right\}$, but it cannot be $+5^{ \pm 1 / 2}$.

\section{The Goeritz matrix and linking form, and a criterion for unknotting number 3}

Let us turn to a formerly known topological approach using the linking form $\lambda$ on $H_{1}\left(D_{K}\right)$. It is a consequence of a result of Montesinos [Mo] and was first stated by Lickorish [Li]. (Note that $D_{K}$ inherits an orientation from $S^{3}$, so that the linking form is given by $+U^{-1}$, where $U$ is a Goeritz [GL] matrix for $K$.)

Proposition 5.1. (Montesinos-Lickorish) If $u(K)=1$, and $\operatorname{det}(K)=D$ then $\left(H_{1}\left(D_{K}\right)\right.$ is cyclic and) there is a generator $g$ of $H_{1}\left(D_{K}\right)$, with $\lambda(g, g)= \pm 2 / D \in$ $\mathbb{Q} / \mathbb{Z}$.

Here $\lambda$ denotes the linking form on $H_{1}\left(D_{K}\right)$ and $u_{ \pm}$denotes the signed unknotting number (see [Tr, CL]), that is, $u_{+}(K)=1$ iff $K$ unknots by switching a positive crossing to the negative. This is the property, to which we found obstructions in $\S 3$, and we will use these obstructions shortly.

There is an extension of the linking form criterion which may be useful when considering signed unknotting number. It appears in a similar form to the one given here in [CL, Proposition 2.1]. (Compare also the proof in Kawauchi's book [Kw, Theorem 11.2.3, pp. 147-8].)

Theorem 5.1. Let $K$ be an unknotting number one knot which can be unknotted by switching a positive crossing to the negative, and $\operatorname{det}(K)=D$. Then 
there is a generator $g$ of $H_{1}\left(D_{K}\right)$ with $\lambda(g, g)=+2 / D \in \mathbb{Q} / \mathbb{Z}$ if $\sigma(K)=0$ and $\lambda(g, g)=-2 / D$ if $\sigma(K)=2$.

Proof. $K$ and the unknot possess Seifert matrices $S$ and $S^{\prime}$ with $S_{i, j}=S_{i, j}^{\prime}$ for $(i, j) \neq(1,1)$ and $S_{1,1}=S_{1,1}^{\prime}+1$. Now $S+S^{T}$ is a representation matrix for $H_{1}\left(D_{K}\right)$, and differs just in the upper left entry from $S^{\prime}+S^{\prime} T$. The determinant $\operatorname{det}\left(S+S^{T}\right)$ is up to sign equal to $\operatorname{det}(K)=D$, and $\operatorname{det}\left(S^{\prime}+S^{\prime} T\right)= \pm 1$. Thus the minor of $S+S^{T}$ obtained by deleting the first row and column from $S+S^{T}$ has determinant $( \pm D \pm 1) / 2$. The homology element $g^{\prime}$ represented by the first row and column then has linking form $\lambda\left(g^{\prime}, g^{\prime}\right)=( \pm D \pm 1) / 2 D$. Set $g=2 g^{\prime}$. Then $\lambda(g, g)= \pm 2 / D$, and hence $g$ is a generator. The important point to notice now is that the sign of $\lambda(g, g)$ depends on whether $\operatorname{det}\left(S+S^{T}\right)$ and $\operatorname{det}\left(S^{\prime}+S^{\prime} T\right)$ have the same sign or not, and that this is equivalent to the signature condition.

Remark 5.1. More generally the proof shows that if $K$ can be turned into some knot $K^{\prime}$ by switching a positive crossing to negative and $\operatorname{det}\left(K^{\prime}\right)=D^{\prime}$, then there is a (not necessarily generating) element $g \in H_{1}\left(D_{K}\right)$ with $\lambda(g, g)=$ $+2 D^{\prime} / D$ if $\sigma(K)=\sigma\left(K^{\prime}\right)$ and $\lambda(g, g)=-2 D^{\prime} / D$ if $\sigma(K)=2+\sigma\left(K^{\prime}\right)$. (This remark will be used later in the proof of Theorem 5.2.)

Remark 5.2. In [CL] an example is given, the $(-9,5,-9)$-pretzel knot, on which Proposition 5.1 does not apply, but Theorem 5.1 excludes one of the possible signs of an unknotting crossing.

The (conjectured) coincidence of the criteria with $Q$ and $\lambda$ for unknotting number 1 (for determinant divisible by 5 ) suggests that further relations between both may exist also for higher unknotting numbers. And indeed, Theorem 5.1, although apparently in practice not much more effective than its unsigned version, can in theory be pushed further, at least in a special case, to prove a new criterion, this time for unknotting number 3 .

Theorem 5.2. If $d=\operatorname{det}(K)$ is a square and has no divisors of the form $4 k+3$, and $\sigma(K)=4$, then $u(K)>2$.

Proof. If $u(K)=2$, then there exists an unknotting sequence $K \rightarrow K^{\prime} \rightarrow \bigcirc$ (latter denoting the unknot). It is known, originally from $[\mathrm{Mu}]$, that for any knot $K$

$$
\operatorname{det}(K) \equiv\left\{\begin{array}{lll}
1(4) & \text { iff } & \sigma(K) \equiv 0(4) \\
3(4) & \text { iff } & \sigma(K) \equiv 2(4)
\end{array} .\right.
$$

Clearly $\sigma\left(K^{\prime}\right)=2$, and hence $d^{\prime}=\operatorname{det}\left(K^{\prime}\right) \equiv 3 \bmod 4$. But then $K$ and $\bigcirc$ are obtained from $K^{\prime}$ by switching crossings of opposite sign (under both of which the signature changes). Now, we apply the argument proving Theorem 5.1 for the crossing switch $K^{\prime} \rightarrow \bigcirc$, and the modified version of this argument given in Remark 5.1 to the crossing switch $K^{\prime} \rightarrow K$ (where in fact we did not involve anything more than the determinant of the Seifert matrices). By combining both, we find some $c \in \mathbb{Z}_{d^{\prime}}$ with $c^{2} \equiv-d \bmod d^{\prime}$. This is impossible, however, whatever $d^{\prime}$ may be, under the condition on $d$ we assumed. (Consider the congruence just 
modulo a prime $4 k+3$ dividing $d^{\prime}$ and use the First supplementary law of number theory for Legendre's symbol.)

Remark 5.3. As in Remark 5.1, the same argument shows that more generally, if $\sigma(K)-\sigma\left(K^{\prime}\right)=4$ and the product $\operatorname{det}(K) \cdot \operatorname{det}\left(K^{\prime}\right)$ is a square and has no divisors of the form $4 k+3$ (which in particular means that $4 \mid \sigma(K), \sigma\left(K^{\prime}\right)$ ), then $K$ and $K^{\prime}$ cannot be interconverted by 2 crossing changes (that is, have distance at least 3 in the sense of Murakami $[\mathrm{Mr}]$ ). For example, $5_{1}$ cannot be made into $4_{1}$ by two crossing changes.

\section{Applications}

If the criterion in Proposition 3.1 applies to both mirror images, or considering one of them is irrelevant (e.g. because the Jones polynomial is self-conjugate or because of the signature), it successfully, and easily, excludes unknotting number one, as for the following knots (most of them due to Nakanishi and KanenobuMurakami), thus shown to have unknotting number two: $7_{4}, 8_{18}, 9_{15}, 9_{17}, 9_{37}$, $9_{40}, 9_{46}, 9_{47}$ and $9_{48}$.

Example 6.1. We briefly demonstrate the example $K=7_{4}$. Its Jones polynomial is $V_{K}(t)=t-2 t^{2}+3 t^{3}-2 t^{4}+3 t^{5}-2 t^{6}+t^{7}-t^{8}$. As in Proposition 3.1 we build $V_{K^{\prime}}$, and obtain $V_{K^{\prime}}\left(e^{\pi i / 3}\right)=2-i \sqrt{3}$, which is not up to sign a power of $i \sqrt{3}$. Thus $V_{K^{\prime}}$ is not the Jones polynomial of a knot $K^{\prime}$, and $7_{4}$ cannot be unknotted by switching a positive crossing to become negative. That $7_{4}$ cannot be unknotted either by switching a negative crossing follows from its signature $\sigma\left(7_{4}\right)=2$.

Even for unknotting number one knots, as $6_{1}$ and $7_{7}$, the criterion in Proposition 3.1 can give non-trivial information. Namely, we can conclude that such knots are unknottable by switching crossings of only one sign (dependingly on how they are obversed), despite having $\sigma=0$. Finally, the criterion recovers beside Lickorish's result [Li] for $7_{4}$ also Traczyk's recent example $10_{67}$ [ $\mathrm{Tr}$ ].

Remark 6.1. K. Murasugi informed me of a paper of Miyazawa [Mi], where he obtained the unknotting numbers of some 10 crossing knots using a formula relating the value $V^{\prime}(-1)$, determinant and the Conway degree-4-Vassiliev invariant (Theorem 8). (Their entries were completed in Kawauchi's tables.) For them all other criteria - Lickorish's, Traczyk's and ours, also apply. Thus it appears that Traczyk's criterion for unknotting number one (and so basically ours, too) is equivalent to Miyazawa's (Miyazawa also obtains a slightly weaker version of Corollary 3.1 in his Corollary 7 ), and they are implied by the linking form condition of Lickorish (see conjectures in $\S 7$ ).

The results we proved in $\S 4$ allow us to settle the unknotting numbers of the knots in (1) as follows: 949 and $10_{103}$, and also $5_{1} \# 4_{1}$, have $u=3$ by Theorem 4.1, and $8_{16}, 10_{86}, 10_{106}, 10_{109}, 10_{116}$, and $10_{121}$ have $u=2$ both by Theorem 4.1 and Proposition 4.1. 
As the simplest special case of this proposition, any knot $K$ with $Q_{K}\left(\frac{\sqrt{5}-1}{2}\right)=$ $+\sqrt{5}$ cannot have unknotting number one. Another possible special case would be a knot $K$ with $\operatorname{det}(K) \equiv \pm 2(5)$ and $Q_{K}\left(\frac{\sqrt{5}-1}{2}\right)=+1$ respectively $\operatorname{det}(K) \equiv$ $\pm 1(5)$ and $Q_{K}\left(\frac{\sqrt{5}-1}{2}\right)=-1$. However, it was shown by Rong [Rn] that such knots do not exist, that is, the sign of $Q_{K}\left(\frac{\sqrt{5}-1}{2}\right)$ for $5 \nmid \operatorname{det}(K)$ is exactly the same as $Q_{K}(2) \bmod 5$. (Rong gives an interpretation of the sign in terms of the Goeritz form.) Therefore, Proposition 4.1 is basically equivalent to Theorem 4.1 for $k=1$.

The unknotting numbers (equal to 2) of $10_{86}, 10_{105}, 10_{106}, 10_{109}, 10_{116}$ and $10_{121}$ are applications of Proposition 5.1. Lickorish's proof of $u\left(7_{4}\right)=2$ also consists in application of this condition, as well as this for 816 , a result of $\mathrm{J}$. R. Rickard whose proof was never published. The disadvantage of this method is that the calculation by hand of $\lambda$ is in general not pleasant. Nevertheless, a nice approach for calculating $\lambda$ was developed by Gordon and Litherland [GL] via the Goeritz matrix $U$. It was carried out on the example $8_{16}$ by Murakami and Yasuhara in [MY] (in slightly generalized form) to give $\lambda\left(g^{\prime}, g^{\prime}\right)= \pm 11 / 35$ for some generator $g^{\prime}$, recovering Rickard's result (as \pm 11 is not twice a square modulo 35). From the Goeritz matrix, $\lambda$ is given by the matrix $U^{-1}$ on the generating set of $H_{1}\left(D_{K}\right)$, on which $U$ gives the relation matrix.

Example 6.2. To make the calculations verifiable, we give as example the data for the knot $K=10_{105}$ obtained from its (unique) 10 crossing diagram. The first four rows give the Goeritz matrix, as relation matrix in $\mathbb{Z}^{4}$, for $H_{1}\left(D_{K}\right)$. The fifth row gives a generator of $H_{1}\left(D_{K}\right)$ (in the basis determined by the Goeritz matrix), preceded by its order. The sixth row gives the Dowker-Thistlethwaite notation, determinant and signature.

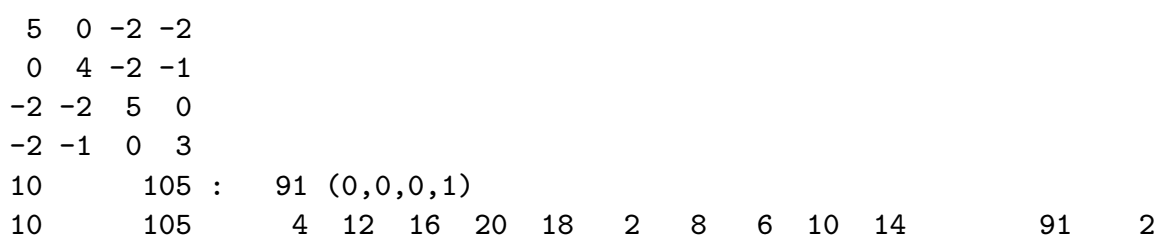

Remark 6.2. In Rolfsen's tables $10_{83}$ and $10_{86}$ are swapped: the Conway notation and Alexander polynomial for each one refers to the diagram of the other. The convention for $10_{86}$ here is that the Conway notations and Alexander polynomials are swapped to fix the discrepancy, and not the diagrams, as in $[\mathrm{Kw}]$ (so our $10_{86}$ is Kawauchi's $10_{83}$ ).

As mentioned, Theorem 5.1 is in general not much more powerful than Proposition 5.1. However, its consequence, Theorem 5.2 again applies to $9_{49}$ and $5_{1} \# 4_{1}$ (but not to $10_{103}$ ) to show $u=3$. Strangely, for all prime knots of $\leq 16$ crossings with $\sigma=4, d=25$ or $d=625$ and 2 torsion numbers in $H_{1}\left(D_{K}\right)$, we had $Q\left(\frac{\sqrt{5}-1}{2}\right)=-5$, so that the $Q$ method excludes $u=2$ as well. This gives further 
hints to an intimate relationship between the sign of the Jones-Rong value of $Q$ and the topology of the double cover of the knot complement.

Nevertheless, the criterion of Theorem 5.2 will clearly give new information for other primes $4 k+1$, which cannot be captured by $Q$. For example, the knot $12_{664}$ of the tables available in Knotscape [HT] has $H_{1}=\mathbb{Z}_{13} \oplus \mathbb{Z}_{13}$ and $\sigma=4$, and hence $u=3$, which cannot be shown by any other method I know of. Another such, possibly more common, example is $6_{3} \# 7_{3}$.

As a summary, beside $8_{16}$ and $9_{49}$, there are several undecided 10 crossing knots in Kawauchi's tables $[\mathrm{Kw}]$, whose unknotting number we found. The (most likely correct) list of prime knots of at most 9 crossings with unknown unknotting number now is: $8_{10}, 9_{29}$ and $9_{32}$ with $u \in\{1,2\}$ and $9_{10}, 9_{13}, 9_{35}$, $9_{38}$ with $u \in\{2,3\}$ (see [Ki, table, p. 49]). Beside the knots so far mentioned, we can complete the entry for $10_{131}$, whose unknotting number is 1 , as show both its diagrams in Knotscape and in Rolfsen's book. The unknotting numbers (so far known to me) of the simplest composite knots are compiled in a table in the appendix.

\section{Conjectures and problems}

In this final section we summarize the unexplained phenomena that came up in our attempts to find unknotting number criteria studying the linking form and the polynomial values, and most of which have been implicitly suggested in our previous discussion. (However, there are also some new ones.) They are all supported by strong empirical evidence. Some of these statements indicate, that the conditions for the unknotting number given by Lickorish [Li], Traczyk $[\mathrm{Tr}]$, and above in this paper seem to imply others, at least in special cases.

The first problem came up in the study of the structure of $H_{1}$ of knots to which Theorem 5.2 is applicable.

Conjecture 7.1. There are no knots with $\sigma=4$ and cyclic $H_{1}$ of order a prime square (i.e., $H_{1}$ is always a double in all such cases). If $\sigma(K)=4$ and $H_{1}=\mathbb{Z}_{5} \oplus \mathbb{Z}_{5}$, then $Q_{K}\left(\frac{\sqrt{5}-1}{2}\right)=-5$ (rather than +5 ).

At least the first part of this conjecture is not true for non-prime squares, even for higher (even) prime powers. There are for example three prime 16 crossing knots with $\sigma=4$ and $H_{1}=\mathbb{Z}_{25} \oplus \mathbb{Z}_{5} \oplus \mathbb{Z}_{5}$. (In the tables of Knotscape they are listed as $16_{902535}, 16_{1344895}$, and $16_{1345031}$, where we index non-alternating knots appended after alternating ones.) By [HNK, Theorem 3.10] there is no $\sigma=4$ knot with determinant 1 . As we saw, the simplest examples supporting the second part of this conjecture are $9_{49}$ and $5_{1} \# 4_{1}$.

Conjecture 7.2. If $K$ has prime determinant $\operatorname{det}(K)=D$ and signature 0 , then there is an element $g \in H_{1}$ with $\lambda(g, g)= \pm 2 / D$.

For $\sigma=2$ this is true, as then $D=4 k+3$. If such $D$ is prime, the multiplicative group $\mathbb{Z}_{D}^{*}$ is cyclic and has no square roots of -1 , so that each residue class $\bmod D$ is either of the form $2 a^{2}$ or $-2 a^{2}$. For $D=4 k+1$ one half of the residue 
classes are of both forms, and the other half of none of them. Latter seem never to occur for $\lambda$ if $\sigma=0$.

Conjecture 7.3. If $K$ has cyclic $H_{1}$ of $\operatorname{order} \operatorname{det}(K)=D$ divisible by 5 , then

$$
Q_{K}\left(\frac{\sqrt{5}-1}{2}\right)=\left\{\begin{array}{lll}
-\sqrt{5} & \text { if } \exists g \in H_{1}: \lambda(g, g)= \pm 2 / D \\
+\sqrt{5} & \text { if } \nexists g \in H_{1}: \lambda(g, g)= \pm 2 / D
\end{array}\right. \text {. }
$$

Conjecture 7.4. Assume $\sigma(K)=+2$, and $H_{1}$ is cyclic. (a) If $V_{K}\left(e^{\pi i / 3}\right)=$ $-i \sqrt{3}$, then there is no $g \in H_{1}$ with $\lambda(g, g)= \pm 2 / D$. (b) If there is no $g \in H_{1}$ with $\lambda(g, g)=-2 / D$, then there is neither a $g \in H_{1}$ with $\lambda(g, g)=+2 / D$.

It seems possible that if $\sigma(K)=+2$ and $H_{1}$ is cyclic of order divisible by 3 , then the conditions $V_{K}\left(e^{\pi i / 3}\right)=-i \sqrt{3}$ and that there is no $g \in H_{1}$ with $\lambda(g, g)= \pm 2 / D$ are equivalent. This motivates the first part of the conjecture. In case $\sigma=2$, I know of no example for which the signed unknotting number information of Theorem 5.1 contradicts that of the signature, but Proposition 5.1 does not apply. This is the origin of the second part (for the case $\sigma=0$, see Remark 5.2).

It seems difficult to generalize Theorem 5.2 in some way. The condition $\sigma=4$ (rather than $\sigma=0$ ) and the lack of divisors $4 k+3$ of the determinant are both necessary (otherwise consider the connected sums of twist knots). How to weaken the squareness condition on the determinant is not clear either, since a computer experiment revealed that all non-square numbers $4 k+1<400$ are realized as determinants of knots with $u=2$ and $\sigma=4$.

The following is a problem on a class of knots where the unknotting number one condition has been strengthened.

Question 7.1. The (reduced alternating) trefoil and figure eight knot diagrams have the property that they unknot by switching any arbitrary crossing. Are they the only (non-trivial ${ }^{1}$ ) knot diagrams with this property? More generally, are for $k>1$ the $(2,2 k \pm 1$ )-torus knot diagrams (with one possible kink in the '-' case) the only diagrams which unknot by switching any arbitrary collection of $k$ crossings?

So far the only observation towards the case $k=1$ is that except for the trefoil diagram any other such diagram must have $\sigma=0$ and $|V(-1)| \equiv 1$ or $5 \bmod 12$.

As a final remark on the $V$ criterion, we mention that there are still some possibilities left open. The most promising way appears to be to consider the Homfly polynomial of the 2-component link arising by smoothing the unknotting crossing and to attack the existence of such a polynomial by the various Vassiliev invariant identities worked out by Kanenobu. We may record an interesting outcome of this (or some similar) idea at a later stage.

\footnotetext{
${ }^{1}$ Diagrams of the unknot with all crossings reducible trivially have this property, too.
} 
Also, one may try to find more special evaluations of the polynomials related to branched cover homology, but the results of [Rn] and [St2] for the $Q$ polynomial (which hold in an analogous form also for $V$, see [JVW]) suggest, that such evaluations, beside the known ones, are very unlikely to exist.

\section{Acknowledgement and credit}

The investigation of this paper was inspired by Traczyk's paper [ $\operatorname{Tr}]$ and his talk on the Conference on Knot Theory "Knots in Hellas, 98". I would also wish to thank to T. Cochran, W. B. R. Lickorish and H. Murakami for helpful discussions on the linking form and to K. Murasugi for telling me of the paper [Mi]. Furthermore, I am grateful to the referee for the careful reading and many useful remarks that improved considerably the exposition.

The paper is written originally in 1999; since then some important developments occurred, in particular the paper of Ozsvath and Szabo [OS], where most unknotting number one problems (including those of 8 and 9 crossings) were finally settled.

\section{References}

[A] N. A'Campo, Generic immersions of curves, knots, monodromy and gordian number, Inst. Hautes Études Sci. Publ. Math. 88 (1998), 151-169.

[Ad] C. C. Adams, Das Knotenbuch, Spektrum Akademischer Verlag, Berlin, 1995 ( The knot book, W. H. Freeman \& Co., New York, 1994).

[BLM] R. D. Brandt, W. B. R. Lickorish and K. Millett, A polynomial invariant for unoriented knots and links, Inv. Math. 84 (1986), 563-573.

[CL] T. D. Cochran and W. B. R. Lickorish, Unknotting information from 4-manifolds, Trans. Amer. Math. Soc. 297(1) (1986), 125-142.

[GL] C. McA. Gordon and R. A. Litherland, On the signature of a link, Invent. Math. 47 (1) (1978), 53-69.

[HNK] F. Hirzebruch, W. D. Neumann and S. S. Koh, Differentiable manifolds and quadratic forms, Lecture Notes in Pure \& Appl. Math. 4, M. Dekker, New York, 1971.

[Ho] C. F. Ho, A polynomial invariant for knots and links - preliminary report, Abstracts Amer. Math. Soc. 6 (1985), 300.

[HT] J. Hoste and M. Thistlethwaite, Knotscape, a knot polynomial calculation and table access program, available at http://www.math.utk.edu/ morwen.

[JVW] F. Jaeger, D. L. Vertigan and D. J. A. Welsh, On the computational complexity of the Jones and Tutte polynomials, Math. Proc. Cambridge Philos. Soc. 108(1) (1990), $35-53$.

[J] V. F. R. Jones, A polynomial invariant of knots and links via von Neumann algebras, Bull. Amer. Math. Soc. 12 (1985), 103-111.

[J2] On a certain value of the Kauffman polynomial, Comm. Math. Phys. 125 (1989), 459-467.

[J3] Hecke algebra representations of of braid groups and link polynomials, Ann. of Math. 126 (1987), 335-388.

[KM] T. Kanenobu and H. Murakami, 2-bridge knots of unknotting number one, Proc. Amer. Math. Soc. 98(3) (1986), 499-502.

[Ka] L. H. Kauffman, State models and the Jones polynomial, Topology 26 (1987), 395-407.

[Km] T. Kawamura, The unknotting numbers of $10_{139}$ and $10_{152}$ are 4, Osaka Journal of Mathematics 35(3) (1998), 539-546.

[Kw] A. Kawauchi, A survey of Knot Theory, Birkhäuser, Basel-Boston-Berlin, 1996. 
[Ki] R. Kirby (ed.), Problems of low-dimensional topology, Geometric topology (Athens, GA, 1993), 35-473, Amer. Math. Soc., 1997, also available on http://math.berkeley.edu/ kirby.

[Ko] T. Kobayashi, Minimal genus Seifert surfaces for unknotting number 1 knots, Kobe J. Math. 6 (1989), 53-62.

[Li] W. B. R. Lickorish, The unknotting number of a classical knot, in "Contemporary Mathematics" 44 (1985), 117-119.

[LM] W. B. R. Lickorish and K. C. Millett, Some evaluations of link polynomials, Comment. Math. Helv. 61 (1986), 349-359.

[Lp] A. S. Lipson, An evaluation of a link polynomial, Math. Proc. Camb. Phil. Soc. 100 (1986), 361-364.

[Mi] Y. Miyazawa, The Jones polynomial of an unknotting number one knot, Topology Appl. 83(3) (1998), 161-167.

[Mo] José M. Montesinos, Surgery on links and double branched covers of $S^{3}$, Knots, groups, and 3-manifolds (Papers dedicated to the memory of R. H. Fox), pp. 227-259. Ann. of Math. Studies 84, Princeton Univ. Press, Princeton, N.J., 1975.

[Mr] H. Murakami, Some metrics on classical knots, Math. Ann. 270(1) (1985), 35-45.

[MY] H. Murakami and A. Yasuhara, 4-genus and 4-dimensional clasp number of a knot, Proc. Amer. Math. Soc. 128(12) (2000), 3693-3699.

$[\mathrm{Mu}] \mathrm{K}$. Murasugi, On a certain numerical invariant of link types, Trans. Amer. Math. Soc. 117 (1965), 387-422.

[Na] Y. Nakanishi, A note on unknotting number, Math. Sem. Notes Kobe Univ. 9(1) (1981), 99-108.

[OS] P. Ozsvath, and Z. Szabo, Knots with unknotting number one and Heegaard Floer homology, preprint math.GT/0401426.

[Ro] D. Rolfsen, Knots and links, Publish or Perish, 1976.

[Rn] Y. W. Rong, The Kauffman polynomial and the two-fold cover of a link, Indiana Univ. Math. J. 40(1) (1991), 321-331.

[Sc] M. Scharlemann, Unknotting number one knots are prime, Invent. Math. 82 (1985), 37-55.

[St] A. Stoimenow, The granny and the square tangle and the unknotting number, Topol. Appl. 117 (2002), 59-75.

[St2] _ Branched cover homology and $Q$ evaluations, Osaka J. Math. 39(1) (2002), 13-21.

[Ta] T. Tanaka, Unknotting numbers of quasipositive knots, Topology and its Applications 88(3) (1998), 239-246.

[Tr] P. Traczyk, A criterion for signed unknotting number, Contemporary Mathematics 233 (1999), 215-220.

[We] H. Wendt, Die Gordische Auflösung von Knoten, Math. Z. 42 (1937), 680-696.

[Zh] X. Zhang, Unknotting number one knots are prime - a new proof, Proc. Amer. Math. Soc. 113 (1991), 611-612.

\section{Appendix I. Tables}

The unknotting number is not a priori additive under connected sum. (That this is true is a long-standing conjecture proved only in the first non-trivial case by Scharlemann [Sc] and possibly even false in general.) Therefore, there seems no reason to favor prime knots in the tabulation of unknotting numbers. For this reason I include a table of the unknotting numbers of composite $\leq 10$ crossing knots as far as feasible to me. 
I adopt the convention of Traczyk [Tr] that the number $x$ followed by $y$ copies of '?' means that the unknotting number is at most $x$, and very likely (or in the case $y=0$ known to be) $x$, but that the values $x-y, \ldots, x-1$ have not yet been excluded. The knots are recorded up to mirroring, taking Thistlethwaite's obversion convention for the factors. This convention can be specified in the relevant cases by saying that if one of $K$ and $! K$ is positively alternating, then always $K$ is taken to be such rather than $! K$. (Positively alternating means that in its alternating diagram all crossings are positive.)

To compile these values, it basically suffices to apply the standard results of $[\mathrm{Mu}, \mathrm{Sc}, \mathrm{We}]$. These methods work except for three knots, where other methods are needed: $7_{4} \# 3_{1}$ and $3_{1} \# 3_{1} \# 4_{1}$ (Traczyk) and $4_{1} \# 5_{1}$ (see above).

\begin{tabular}{|l|l||l|l||l|l||l|l|}
\hline$K$ & $u(K)$ & $K$ & $u(K)$ & $K$ & $u(K)$ & $K$ & $u(K)$ \\
\hline \hline $3_{1} \# 3_{1}$ & 2 & $3_{1} \# ! 6_{1}$ & 2 & $3_{1} \# ! 7_{2}$ & 2 & $3_{1} \# ! 7_{7}$ & 2 \\
$3_{1} \# ! 3_{1}$ & 2 & $3_{1} \# 6_{2}$ & 2 & $3_{1} \# 7_{3}$ & 3 & $4_{1} \# 6_{1}$ & 2 \\
$3_{1} \# 4_{1}$ & 2 & $3_{1} \# ! 6_{2}$ & 2 & $3_{1} \# ! 7_{3}$ & $3 ?$ & $4_{1} \# 6_{2}$ & 2 \\
$3_{1} \# 5_{1}$ & 3 & $3_{1} \# 6_{3}$ & 2 & $3_{1} \# 7_{4}$ & 3 & $4_{1} \# 6_{3}$ & 2 \\
$3_{1} \# ! 5_{1}$ & $3 ?$ & $4_{1} \# 5_{1}$ & 3 & $3_{1} \# ! 7_{4}$ & $3 ?$ & $5_{1} \# 5_{1}$ & 4 \\
$3_{1} \# 5_{2}$ & 2 & $4_{1} \# 5_{2}$ & 2 & $3_{1} \# 7_{5}$ & 3 & $5_{1} \# ! 5_{1}$ & $4 ? ?$ \\
$3_{1} \# ! 5_{2}$ & 2 & $3_{1} \# 3_{1} \# 4_{1}$ & 3 & $3_{1} \# ! 7_{5}$ & $3 ?$ & $5_{1} \# 5_{2}$ & 3 \\
$4_{1} \# 4_{1}$ & 2 & $3_{1} \# ! 3_{1} \# 4_{1}$ & $3 ?$ & $3_{1} \# 7_{6}$ & 2 & $5_{1} \# ! 5_{2}$ & $3 ?$ \\
$3_{1} \# 3_{1} \# 3_{1}$ & 3 & $3_{1} \# 7_{1}$ & 4 & $3_{1} \# ! 7_{6}$ & 2 & $5_{2} \# 5_{2}$ & 2 \\
$3_{1} \# 3_{1} \# ! 3_{1}$ & 3 & $3_{1} \# ! 7_{1}$ & $4 ? ?$ & $3_{1} \# 7_{7}$ & 2 & $5_{2} \# ! 5_{2}$ & 2 \\
$3_{1} \# 6_{1}$ & 2 & $3_{1} \# 7_{2}$ & 2 & & & & \\
\hline
\end{tabular}

Graduate School of Mathematical Sciences, University of Tokyo, 3-8-1, Komaba, TOKYO 153-8914, JAPAN

E-mail address: stoimeno@ms.u-tokyo.ac.jp

$U R L:$ http://www.ms.u-tokyo.ac.jp/〜stoimeno/ 